
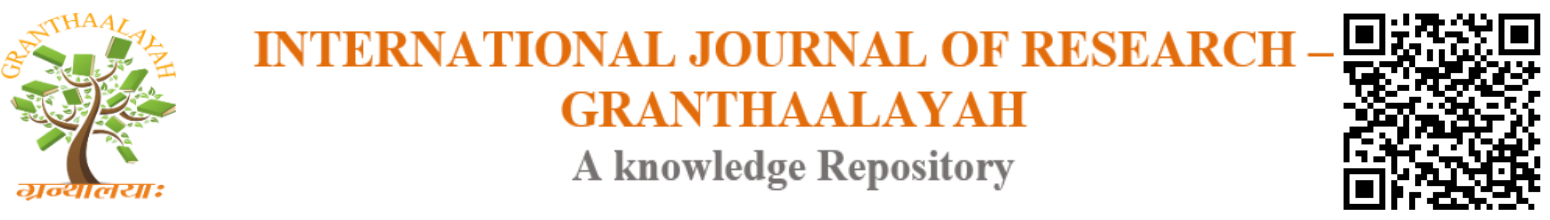

Science

\title{
A STUDY ON LOW CONTRAST VIDEO ENHANCEMENT BASED ON RETINEX
}

\author{
Qiming Wang ${ }^{* 1}$, Hesheng Shi ${ }^{1}$, Zhiqing Zhou ${ }^{1}$ \\ ${ }^{* 1}$ College of Information Engineering, Pingdingshan University, Pingdingshan, Henan 467002, \\ China
}

\begin{abstract}
This paper presents a video adaptive enhancement method based on Retinex illumination theory. The bidirectional equalization algorithm is adopted to make the gray distribution more uniform and to suppress the noise to a certain extent. The self-adaptive enhancement function stretches the radiation component and the overall component and improves the global and local visual effects. In the splicing process of the reflection component, the self-adaptive weighted method is effective to solve the fuzzy block effect, and the introduction of the similarity between frames validly improves the processing efficiency of the algorithm. Experiments have demonstrated that the proposed method can effectively deal with low-contrast video, and the enhancement effect is obvious, fast and effective.
\end{abstract}

Keywords: Fuzzy Matching; Motion Compensation; Zernike Moment; Correlation Analysis.

Cite This Article: Qiming Wang, Hesheng Shi, and Zhiqing Zhou. (2018). "A STUDY ON LOW CONTRAST VIDEO ENHANCEMENT BASED ON RETINEX." International Journal of Research - Granthaalayah, 6(11), 27-34. 10.29121/granthaalayah.v6.i11.2018.1085.

\section{Introduction}

In space cooperation, a substantial amount of information needs to be taken from the image information of the moving objects in the video. In particular, in the final stage of spatial cooperation, the relative position information between the subsystems should be acquired by CCD and other sensors. The acquisition, transmission, processing efficiency and quality of the moving target images play a vital role in accurately capturing the position, velocity and attitude information of moving objects and guaranteeing the smooth reoccurrance of the target movement in space cooperation (Caspi et al., 2006). But in reality, physical devices, shooting ambient light and other objective circumstances will lead to underexposure in some areas of pictures and videos, thereby seriously affecting the quality of the video. These data is often not able to be directly used for postprocessing, and contrast enhancement technology can address this problem (Shechtman et al., 2012). The traditional methods include histogram equalization algorithm, logarithmic stretching algorithm and the derivative methods of these algorithms (Liu and Sun, 2011; Protter et al., 2009). The parameter setting of these algorithms or an excessive number of users cause difficulties in 
practice and a limited practicality of the algorithms. In recent years, Retinex lighting theory has been proposed and applied to enhance contrast, and favorable results are achieved (Chen and Lorenz, 2012). The algorithm proposed in this paper builds on the Retinex, the logarithmic extensional theory and the statistical information of image gray scale, automatically adjusts the method parameters, applies the similarity between video frames to make better use of preinformation, and improves the processing efficiency. The improved logarithmic tensile processing protects and effectively enhances the detailed information in the underexposed area without overenhancement, and validly elevates the quality of the video.

\section{RETINEX Lighting Theory}

Land has introduced Retinex theory as a human visual system (HVS) to explain color constancy. Human visual model is sensitive to the reflection information on the target surface (Tomasi et al., 2012). The proposed model expresses the image as the product of each pixel of the irradiation component and the target component. Retinex's formulaic description of the image is as below.

$$
S(x, y)=L(x, y){ }^{*} R(x, y)
$$

In the formula, $S(x, y)$ represents the image received by the camera. $L(x, y)$ indicates the illuminated light. $\mathrm{R}(\mathrm{x}, \mathrm{y})$ signifies the reflection characteristic of the object itself.

According to Retinex theory, the light information received by human eyes is the product of the irradiation component and the reflection component. The intensity of the light will affect the light and shade of the entire image, and the original appearance of the scene is determined by the reflection characteristics (Kim, 1997). The purpose of this theory is to estimate the radiation component according to the received image, remove it and derive the reflection component, so as to restore the original appearance of the scene. In general, the obtained image is subjected to Gaussian convolution to obtain the irradiation component. Its Gaussian kernel function is as follows:

$$
F(x, y)=C^{*} \exp \left(-\frac{x^{2}+{ }^{y 2}}{2 * \sigma^{2}}\right)
$$

Specifically, $\sigma$ is the scale of the convolution function and $\mathrm{C}$ satisfies $\iint F(x, y) d x d y=1$. In the end, reflection component is calculated as follows:

$$
\log R(x, y)=\log S(x, y)-\log S(x, y) * F(x, y)
$$

Where * is the convolution calculation. Formula (3) is taken exponentially to obtain the reflection component $\mathrm{R}(\mathrm{x}, \mathrm{y})$.

\section{Propose the Video Contrast Enhancement Algorithm}

In this paper, the Retinex enhancement method is improved, and a logarithmic stretching method is introduced in the process of solving the reflection component. Also, a video adaptive contrast enhancement algorithm is proposed.

- Improved Bidirectional Equalization Algorithm 
The essential feature of bidirectional equalization algorithm is that the pixels can be uniformly spread on $[0,255]$. Its basic idea is to calculate the non-zero grayscale in the grey level histogram, and the number of the obtained actual gray levels is Ne. Subsequently, grayscale is sorted and arranged in an evenly spaced way within the range of [0.255]. The conversion function is as follows:

$T_{k}=j * \frac{255}{N e} \quad j=0,1,2, \ldots, k$

However, the disadvantage of bi-directional equalization is the method of calculating the non-zero gray level. As long as the number of pixels in the gray scale is not zero, it should be taken into account. In this way, when the variation range of grayscale is large, the pixels cannot be well uniformly spaced with good adaptability. Therefore, in this paper, when the non-zero gray level is counted, the threshold $\mathrm{Nr}$ is set, which will be taken into account only in the case of $\mathrm{Ni}>\mathrm{Nr}$. If the number of pixels is less than the threshold value $\mathrm{Nr}$, they are directly discarded, and the algorithm will filter noise points to a certain extent at the same time.

\section{- Improvements to the Retinex Method}

The Retinex contrast processing method abstracts the image information into an illumination model and a reflection model. The acquisition of the illumination model can be approximated by the convolution of the image. Because Gaussian filter emphasizes the boundaries between those regions that are in a relatively gradual variation, which is practical in a number of applications. Therefore, Gaussian low-pass filter is applied to make an approximate evaluation on the irradiation component. Furthermore, the reflection component is derived from the irradiation component.

In order to make the image have a better contrast, it is necessary to correct the irradiation component and to enhance the tensile image grayscale that is self-adaptable to the function (Wang et al., 1999). The selected nonlinear function is

$$
F(i)=\left\{\begin{array}{cl}
0 & i<L-k^{*} R \\
\frac{i-\left(L-k^{*} R\right)}{2 k^{*} R} & L-k^{*} R \leq i \leq L+k^{*} R \\
1 & i>L+k^{*} R
\end{array}\right.
$$

In the formula, $\mathrm{L}$ and $\mathrm{R}$ represent the mean value and the standard deviation value of the gray scale, respectively. This form of transformation can make the image gray value reach the maximum stretching effect near its mean value, and also can avoid the phenomenon of over-enhancement while ensuring the colors.

Reflection component indicates the details of the image, so adjusting the value of the reflection component can enhance the local details of the image. Since a very large or very small value of the pixel corresponds to the sharp region of the images, these areas generally produce better visual effects. The reflection components of the transformed flat area $\mathrm{r}$ should be adjusted, and the values of the flat areas are relatively close. The degree of adjustment of reflection component is not uniform. The following enhancement function satisfies the requirement as below. 


$$
s(r)= \begin{cases}e_{1}+\left(t-e_{1}\right) *\left(\frac{r-e_{1}}{t-e_{1}}\right)^{k} & e_{1} \leq r<t \\ e_{2}-\left(e_{2}-t\right) *\left(\frac{e_{2}-r}{e_{2}-t}\right)^{k} & t \leq r \leq e 2\end{cases}
$$

Specifically, e1=min $(r) ; e 2=\max (r) ; t=(e 1+e 1) / 2$. These parameters can be calculated according to the gray value of the image, so its adaptability is stronger.

In the splicing process of reflective component, due to the size difference of the gray value of the local pixel, the enhanced image demonstrates a significant fuzzy block effect. Therefore, in the process of splicing, the self-adaptive weight matrix is applied to elevate the proportion of the pixel gray value approximating the average gray value. These pixels represent the ideal pixels in the local area, so the application of this method eliminates the impact of gray difference and achieves the purpose of weakening block effect.

\section{- Frame-to-frame Difference Method Locally Adjusts the Brightness of Motion Area}

Concerning video enhancement, frame-to-frame information can also be adopted to increase image brightness. An algorithm based on the adjacent frame difference is often used to detect the moving image sequence and the moving target, which is applied to brightness processing to determine the similarity of the adjacent frames (Fayad et al., 2002). The basic principle of the adjacent frame difference method is to subtract the gray values of the pixels corresponding to two adjacent frames. In case of a small change in the ambient brightness, if the difference of the corresponding pixel gray-scale is quite small, the scene is regarded to be still; if the gray scale of some image region largely changes, it is regarded to be caused by the moving objects in the image (Gonzalez and Wintz, 1992). Its outstanding features include simple implementation and fast operation, and the detection effects are favorable in most cases. It is often adopted in occasions that hold relatively high real-time requirements. In terms of the two consecutive frames in the image sequence, the image correlation is fairly strong, thereby introducing the related concepts of frame-to-frame.

$$
R=\frac{\sum_{m} \sum_{n}\left(I_{i_{m m}}-\bar{I}_{i}\right)\left(I_{j_{m m}}-\overline{I_{j}}\right)}{\left(\sum_{m} \sum_{n}\left(I_{i_{m m}}-\bar{I}_{i}\right)^{2} * \sum_{m} \sum_{n}\left(I_{j m}-\overline{I_{j}}\right)^{2}\right)^{1 / 2}}
$$

In the formula, $\bar{I}$ is the mean value of each frame. Ii and $\mathrm{Ij}$ are the images of two different frames. A larger value of $\mathrm{R}$ indicates a higher similarity of two frames of images.

In this paper, if the correlation of two consecutive frames is very high, it states that they hold a high consistency in time and space, and there exists a corresponding relation between the adjacent two frames in terms of brightness. On the other hand, it indicates a low similarity, incoherent motion and a little corresponding relation of brightness. Therefore, similar thresholds are set. When the threshold is exceeded, it is considered that the similarity of the adjacent two frames is high, and the processing result of the previous frame can be regarded as the result of that of the previous frame, which effectively boosts the processing efficiency of the video. 


\section{Experimental Results and Analysis}

The videos employed in the experiment respectively demonstrate the spacecraft and the standard library. The above-proposed improvement method is adopted to process the videos. In the convenience to illustrate the experiment results, only the processing effect of some frames is demonstrated, and the traditional HE and zm_Retinex methods are applied for a comparative analysis. The processing results are indicated in the following figure.
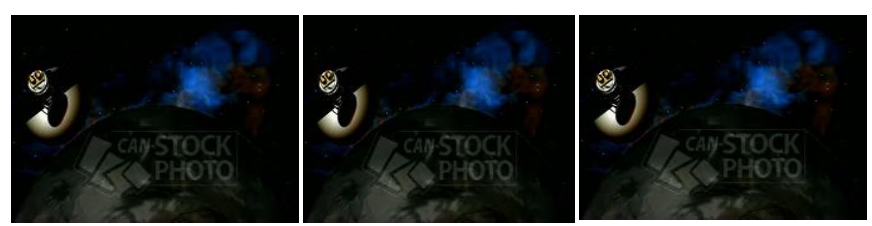

The 13-th Frame The 25-th Frame The 47-th Frame

Figure 1: Spacecraft sequence

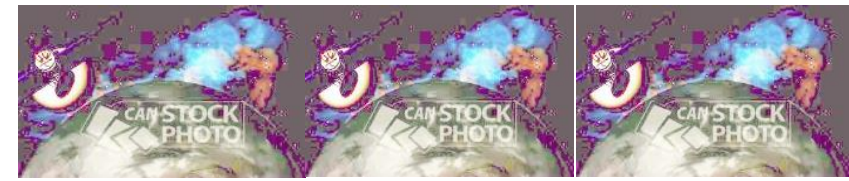

Figure 2: HE enhancement algorithm

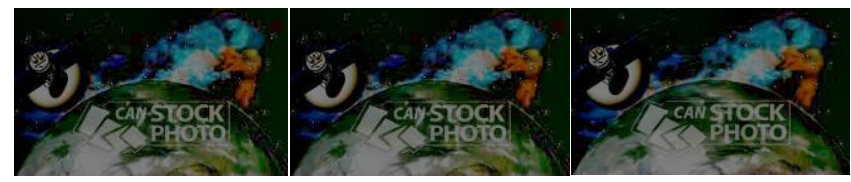

Figure 3: zm_Retinex enhancement method

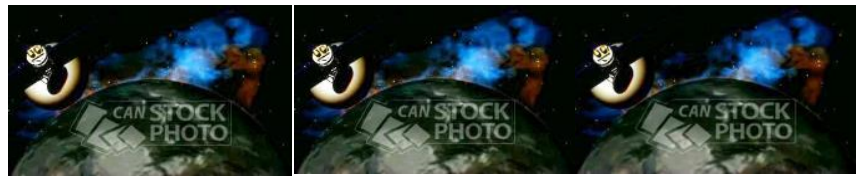

Figure 4: Method in this paper

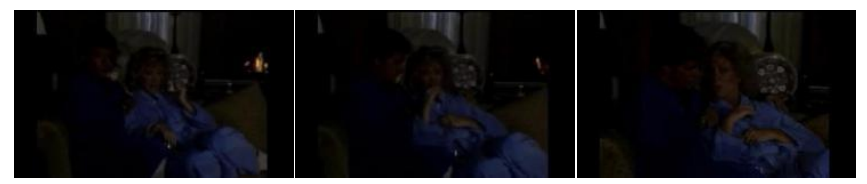

The 263-th Frame The 272-th Frame The 344-th Frame

Figure 5: Standard video

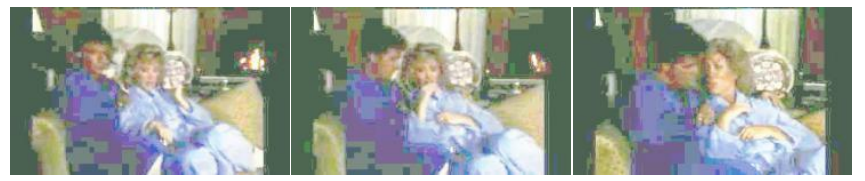

Figure 6: HE enhancement algorithm 


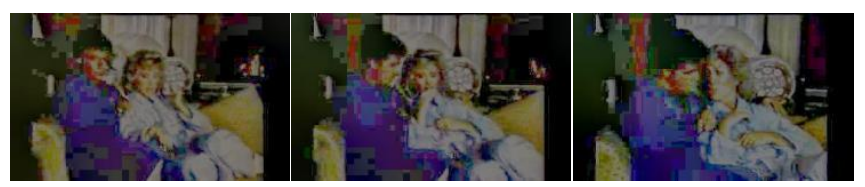

Figure 7: zm_Retinex enhancement method

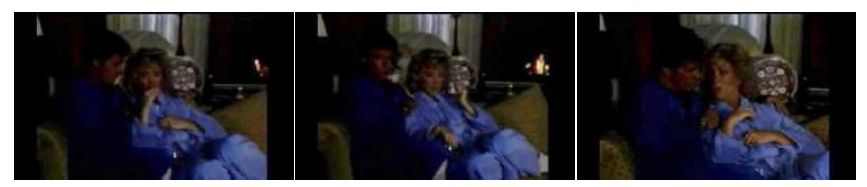

Figure 8: Method in this paper

The experimental results show that the method proposed in this paper produces a better enhancement effect than zm_Retinex and HE. According to the two comparison experiments, HE method has a wide range of obvious over-enhancement, overly bright image on the whole and seriously distorted colors. At the same time, it is indicated that the enhancement effect of zm_Retinex is much better than that of HE, but the local fuzzy block effect occurs in the processing results and the image color is distorted while the brightness is raised. This proposed method suppresses noise amplification by means of the bidirectional equalization algorithm, and the selfadaptive enhancement function elevates the overall brightness while improving the local detail information and the adaptive splicing strategy of the reflection component. The fuzzy block effect of Retinex is overcome, and the enhancement effect of the image is more apparant. In the two groups of contrast experiments, subjectively speaking, it is clearly seen that the enhanced results in this paper generate richer details, more clear images, better color and better visual effects.

In terms of an objective evaluation on the indicators, the information entropy indicators in Table 1 and Table 2 clearly illustrate that the values in this paper are higher than that of $\mathrm{HE}$ and zm_Retinex, indicating that the reconstructed image has more clear details. As far as the brightness indicator is concerned, zm_Retinex has a slightly higher value than this proposed method, which is caused by the over-enhancement in the zm_Retinex method. Considering the standard deviation indicator, the method of this paper generates a more even gray distribution in the image than zm_Retine, with more vivid colors and more clear visual effects. As a result of this method, a bidirectional equalization strategy and a self-adaptive enhancement function are applied to remove the influence of fuzzy blocks.

Table 1: Comparisons of Spacecraft Contrast Enhancement Indicators (25 frames)

\begin{tabular}{|l|l|l|l|}
\hline Brightness enhancement method & Lightness & Standard deviation & Entropy \\
\hline Original image & 128.3880 & 10.8176 & 7.1440 \\
\hline HE & 180.6242 & 10.6794 & 7.2429 \\
\hline zm_Retienx & 161.6064 & 10.5296 & 7.4001 \\
\hline Algorithm in this paper & 159.6967 & 11.0007 & 7.5738 \\
\hline
\end{tabular}


Table 2: Comparisons of Standard Library Contrast Image Enhancement Indicators (263 frames)

\begin{tabular}{|l|l|l|l|}
\hline Brightness enhancement method & Lightness & Standard deviation & Entropy \\
\hline Original image & 24.0348 & 3.3557 & 3.4138 \\
\hline HE & 82.6994 & 10.4679 & 3.3750 \\
\hline zm_Retinex & 64.8701 & 2.2292 & 3.4446 \\
\hline Algorithm in this paper & 63.7699 & 3.9670 & 4.0702 \\
\hline
\end{tabular}

\section{Conclusions}

This paper has proposed a video contrast enhancement method based on Retinex, and the bidirectional equalization algorithm makes the gray distribution of the image more uniform, and threshold setting removes the impact of noise to a certain extent. On the basis of Retinex theory, the self-adaptive tensile enhancement is respectively conducted on irradiation component and reflection component, thereby upgrading the overall brightness and enhancing the quality of local details. In the splicing process of the reflection component, a self-adaptive threshold matrix is introduced to eliminate the fuzzy block effect of Retinex, and the visual effect of the enhanced image effect is better. In the process of video processing, the similarity between frames greatly improves the efficiency of the algorithm and provides space cooperation with high-quality video data.

\section{Acknowledgment}

This work is supported by Science and technology project of Henan Province in 2016 (Item No.162102310248), Science and technology project of Henan Province in 2017 (Item No.172102210118).

\section{References}

[1] Caspi Y., Simakov D., Irani M. (2006). Feature-Based Sequence to Sequence Matching, International Journal of Computer Vision, Vol. 68, No. 1, pp. 53-64.

[2] Shechtman E., Caspi Y., Irani M. (2012). Increasing Space-Time Resolution in Video, European Conference on Computer Vision, Vol. 22, No. 7, pp. 1023-1027

[3] Liu C., Sun D. (2011). A Bayesian approach to adaptive video super resolution, Computer Vision and Pattern Recognition, Vol. 42, No. 7, pp. 209-216

[4] Protter M., Elad M., Takeda H., Milanfar P. (2009). Generalizing the Nonlocal-Means to SuperResolution Reconstruction, IEEE Trans on Image Processing, Vol. 18, No. 1, pp. 36-51.

[5] Chen K., Lorenz D. (2012). Image Sequence Interpolation Based on Optical Flow, Segmentation, and Optimal Control, Vol. 21, No. 3, pp. 1020-1030.

[6] Tomasi M., Vanegas M., Barranco F., Daz J., Ros E. (2012). Massive Parallel-Hardware Architecture for Multiscale Stereo, Optical Flow and Image-Structure Computation, IEEE Transactions on Circuits \& Systems for Video Technology, Vol. 22, No. 2, pp. 282-294.

[7] Kim Y.T. (1997). Contrast enhancement using brightness preserving bi-histogram equalization, IEEE Transactions on Consumer Electronics, Vol. 43, No. 1, pp. 1-8. 
[8] Wang Y., Chen Q., Zhang B. (1999). Image enhancement based on equal area dualistic sub-image histogram equalization method, IEEE Transactions on Consumer Electronics, Vol. 45, No. 1, pp. 68-75.

[9] Fayad L.M., Jin Y., Laine A., Berkmen Y., Pearson G., Freedman B., Van H.R. (2002). Chest CT window settings with multiscale adaptive histogram equalization: pilot study, Radiology, Vol. 233, No. 3, pp. 845-852.

[10] Gonzalez R.C., Wintz P. (1992). Digital image processing (2nd ed.), Addison-Wesley Longman Publishing Co., Inc., Vol. 6, No. 5, pp. 85-103.

[11] Cui Y.F., Zhang Q.X., Han W.G., Zhao D.D. (2016). Fatigue life analysis of spur gears with precise tooth profile surfaces, Mathematical Modelling of Engineering Problems, Vol. 3, No. 2, pp. 81-86. DOI: 10.18280/mmep.030207

[12] hang L., Zhang T.D., Gao R., Tang D.Y., Yang J.Y., Fu T.L., Zhan Z.L. (2016). Phenol adsorption property of high specific surface areas biomass based porous carbon materials, Mathematical Modelling of Engineering Problems, Vol. 3, No. 4, pp. 157-161. DOI:10.18280/mmep.030401

[13] Li Y.J. (2016). The mathematical forecast model of Shandong GDP and the analysis of its influencing factors, Advances in Modelling and Analysis A, Vol. 53, No. 2, pp. 150-163

[14] Baldayrou E., Rouge M., Merelle S., Merelle G., Devevey A. (2016). Introducing fun karaoke workshop within a therapeutic education program: clinical findings inparkinsonian persons, Modelling, Measurement and Control C, Vol. 77, No. 2, pp. 29-39.

[15] Wang J.T. Yu W.L., Wang T., Wang Y.L., Gao Y.L. (2015). First-principles study on the thermodynamic defect and crystal structure of U-12.5 at\% Nb alloy, International Journal of Heat and Technology, Vol. 33, No. 1, pp. 175-180. DOI:10.18280/ijht.330124

\footnotetext{
*Corresponding author.

E-mail address: wqm8157@ 126.com
} 